\title{
ENSAIO DE MICROINFILTRAÇÃO: REVISÃO DA LITERATURA
}

\section{MICROLEAKAGE TEST: A REVIEW}

\author{
Mayra Fidelis Zamboni Quitero* \\ Anely Oliveira Lopes* \\ Adriana Bona Matos"*
}

\begin{abstract}
RESUMO
A durabilidade das restaurações diretas está intimamente relacionada ao vedamento do ângulo cavossuperficial de preparos cavitários. Apesar de toda a evolução pela qual os sistemas adesivos e materiais restauradores têm passado, ainda não há perfeito selamento da interface dente-restauração. Logo, há necessidade de aprimorar as técnicas e os materiais restauradores e adesivos, com o objetivo principal de minimizar a formação de fendas marginais e consequente microinfiltração. Analisando a literatura sobre o tema, observa-se que existem diferentes metodologias para a realização do ensaio de microinfiltração. O objetivo deste trabalho é apresentar possíveis fatores que podem interferir nos resultados dese teste. Para tal fim, foi realizada uma revisão bibliográfica detalhada do tema. Conclui-se que a metodologia mais frequentemente encontrada na literatura utiliza dentes humanos com preparos cavitários de Classe $\mathrm{V}$, como método de envelhecimento a ciclagem térmica, o corante azul de metileno e a leitura dos resultados feita através da análise qualitativa.
\end{abstract}

Descritores: Infiltração dentária • Metodologia.

\section{ABSTRACT}

The durability of direct restorations is closely related to the superficial angle of dental cavities. Despite all the changes which the adhesive systems and restorative materials have passed, the sealing of the tooth / restoration interface is not perfect yet. Therefore, the improvement of the techniques and restorative materials is important to minimize the formation of marginal gaps and consequent microleakage. Reviewing the literature on this subject, it was noted that there are different methodologies for conducting the microleakage test. The objective of this study is to discuss possible factors that may affect results of this test. It was concluded that the methodology most commonly found in the literature uses human teeth with cavity preparations of Class $\mathrm{V}$; the thermal cycling; the methylene blue dye; and the qualitative analysis to avaliate the results.

Descriptors: Dental Leakage • Methodology.

* Mestranda em Dentística pela Faculdade de Odontologia da Universidade de São Paulo - FOUSP. E-mail: mayra.quitero@usp.br

** Mestranda em Dentística pela Faculdade de Odontologia da Universidade de São Paulo - FOUSP. E-mail: anely.lopes@usp.br

*** Professora Doutora associada do Departamento de Dentística da Faculdade de Odontologia da Universidade de São Paulo - FOUSP. E-mail: bona@usp.br 
QUITERO MFZ

LOPES AO

MATOS $A B$

ENSAIO DE MICROINFILTRAÇÃO: REVISÃO DA LITERATURA

REV. ODONTOL. UNIV. CID. SÃO

PAULO

2012; 24(2): $123-$

33. MAIO-AGO:

\section{N T RO DUÇÃO}

A durabilidade das restaurações diretas está intimamente relacionada ao vedamento do ângulo cavossuperficial de preparos cavitários. Apesar de toda a evolução pela qual os sistemas adesivos e materiais restauradores têm passado, ainda não há perfeito selamento da interface dente-restauração. Logo, há necessidade de aprimorar as técnicas e os materiais restauradores e adesivos, com o objetivo principal de minimizar a formação de fendas marginais e consequente microinfiltração, definida como a passagem, clinicamente não detectável, de bactérias, fluidos, moléculas ou íons entre a parede cavitária e o material restaurador (Kidd ${ }^{1}$, 1976). É um fenômeno que envolve difusão e registra a relação dinâmica entre a estrutura dentária e o material restaurador (Trowbridge ${ }^{2}$, 1987).

Analisando a literatura sobre o tema, observa-se que existem diferentes metodologias para a realização do ensaio de microinfiltração. O objetivo deste trabaIho é apresentar possíveis fatores que podem interferir nos resultados desse teste. Para tal fim, foi realizada uma revisão bibliográfica detalhada do tema.

\section{REVISÃO E DISCUSSÃO DA LITERATURA}

\section{- Seleção do substrato de teste}

Os trabalhos de microinfiltração localizados na literatura utilizam a) dentes humanos (Akgungor e Akkayan 3 , 2006, Attam et al. ${ }^{4}, 2009$, Attar et al. ${ }^{5}, 2008$, Brackett et al. ${ }^{6}, 2004$, Casagrande et al. ${ }^{7}$, 2005, Celiberti e Lussi ${ }^{8}, 2005$, Cenci et al. ${ }^{9}, 2005$, Chuang et al. ${ }^{10}, 2001$, Delme et al. ${ }^{11}, 2005$, Elgalaid et al. ${ }^{12}$, 2004, Fugaro $^{13}, 2010$, Giachetti et al. ${ }^{14}, 2008$, Hegde et al. ${ }^{15}, 2009$, Helvatjoglu-Antoniades et al. ${ }^{16}, 2004$, Kucukesmen e Sonmez ${ }^{17}$, 2008, MR et al. ${ }^{18}, 2010$, Manhart et al. ${ }^{19}$, 2001, Moshonov et al. ${ }^{20}$, 2005, Nilgun Ozturk et al. ${ }^{21}, 2004$, Pradelle-Plasse et al. ${ }^{22}, 2004$, RP. et al. ${ }^{23}, 2009$, Yazici et al. ${ }^{24}, 2001$, Senawongse et al...25, 2010, Sharma et al. ${ }^{26}, 2009$, Sung et al. ${ }^{27}, 2004$, Wahab et al. ${ }^{28}, 2003$, Yavuz et al. ${ }^{29}, 2006$, Yazici et al. ${ }^{30}, 2008$, Youssef et al. ${ }^{31}, 2006$, Ziskind et al. ${ }^{32}$, 2005) b) e dentes bovinos
(Yavuz et al. ${ }^{29}, 2006$, Braga et al. $.^{33}, 2006$, Calheiros et al.34, 2004, Sassi et al..$^{35}$, 2008, Kawaguchi et al.36, 2004, Kubo et al. ${ }^{37}, 2004$, Nakamichi et al. ${ }^{38}, 1983$, Silva Santana et al. ${ }^{39}, 2009$ ).

Por um lado a utilização de dentes bovinos tem sido apontada como vantajosa Brackett et al.40, 1998, Brackett et al. ${ }^{41}$, 1997 pela facilidade de aquisição, similaridade da época de extração Schmalz et al. ${ }^{42}, 2001$ e menor variabilidade da permeabilidade dentinária, quando comparada aos dentes humanos (Schmalz et al. ${ }^{42}$, 2001). Por outro lado, as desvantagens apresentadas devem ser levadas em consideração, tais como menor resistentência aos ácidos Nakabayashi e Pashley ${ }^{43}$, (2000), alto grau de infiltração e baixos valores de resistência de união Retief ${ }^{44}, 1991$ devido à densidade dos túbulos dentinários bovinos ser significantemente maior (Schilke et al. $\left.{ }^{45}, 2000\right)$. Há, ainda, uma terceira vertente que relata que a composição e ultraestruturas de tags de resina em dentina bovina e humana condicionadas são similares in vitro Titley et al. ${ }^{46},(1995)$, sendo a dentina coronária bovina considerada por alguns autores como substituta de dentes humanos em estudos de adesão e microinfiltração (Efes $\left.{ }^{47}, 2003\right)$.

Diante das opiniões divergentes encontradas na literatura, acredita-se que seria mais adequado utilizar dentes bovinos para treinamento de metodologia e realização de estudos piloto, enquanto que, quando da obtenção dos dados da pesquisa, a utilização da dentina humana é preferível Nakabayashi e Pashley ${ }^{43}$, (2000), desde que todos os aspectos éticos da sua utilização sejam respeitados.

Quanto ao tamanho da amostra, observou-se que a maioria dos artigos revisados utilizou entre 40 e 110 unidades experimentais para compor a amostra (n total). Já em relação ao $\mathrm{n}$ por grupo, algumas diferenças foram detectadas. Predominantemente, trabalhos utilizaram número de repetições por grupo $(n)$ igual a dez (Attam et al. ${ }^{4}, 2009$, Cenci et al. ${ }^{9}, 2005$, MR et al. ${ }^{18}, 2010$, Manhart et al. ${ }^{19}, 2001$, Moshonov et al. $.^{20}, 2005$, RP. et al. ${ }^{23}, 2009$, Sharma et al. $.^{26}, 2009$, Sung et al. ${ }^{27}, 2004$, Yazici et al. $.^{30}, 2008$, Youssef et al. ${ }^{31}, 2006$, Ziskind et al. ${ }^{32}, 2005$, Fleming et al..8, 
2005), cinco (Helvatjoglu-Antoniades et al. ${ }^{16,}$ 2004, Pradelle-Plasse et al. ${ }^{22}$, 2004), quinze (Attam et al. ${ }^{4}, 2009$, Hegde et al. ${ }^{15}$, 2009, Nilgun Ozturk et al. ${ }^{21}$, 2004, Yazici et $a ._{.}^{24}, 2001$ ) ou vinte (Celiberti e Lussi ${ }^{8}$, 2005, Chuang et al. ${ }^{10}, 2001$, Wahab et al. $\left.{ }^{28}, 2003\right)$. Considera-se que o número de dentes a serem utilizados em cada grupo está relacionado ao número de fatores de variação presente no estudo, mas acreditamos que dez dentes por grupo seja um n suficiente para obtenção de resultados confiáveis, desde que o operador seja treinado para a metodologia em questão.

Quanto à procedência dos dentes humanos, observou-se que a maioria dos artigos relata que eles eram recém-extraídos por motivos ortodônticos ou por necessidade cirúrgica, no caso de terceiros molares (Akgungor e Akkayan³, 2006, Attam et al. ${ }^{4}, 2009$, Attar et al. ${ }^{5}, 2008$, Brackett et al. ${ }^{6}, 2004$, Casagrande et al. ${ }^{7}$, 2005, Celiberti e Lussi ${ }^{8}, 2005$, Cenci et al. ${ }^{9}, 2005$, Chuang et al. ${ }^{10}, 2001$, Delme et al. ${ }^{11}, 2005$, Elgalaid et $\mathrm{a}^{13}, 2010$, Giachetti et al. ${ }^{14}$, 2008, Hegde et al. ${ }^{15}, 2009$, Helvatjoglu-Antoniades et al. ${ }^{16}, 2004$, Kucukesmen e Sonmez ${ }^{17}, 2008$, MR et al. ${ }^{18}$, 2010, Manhart et al. ${ }^{19}$, 2001, Moshonov et al. $.^{20}, 2005$, Nilgun Ozturk et al. ${ }^{21}, 2004$, Pradelle-Plasse et al. ${ }^{22}, 2004$, RP. et al. ${ }^{23}$, 2009, Yazici et al. ${ }^{24}, 2001$, Senawongse et al. ${ }^{25}, 2010$, Sharma et al. ${ }^{26}, 2009$, Sung et al. ${ }^{27}, 2004$, Yavuz et al. ${ }^{29}, 2006$, Yazici et al. ${ }^{30}, 2008$, Youssef et al. ${ }^{31}, 2006$, Ziskind et al. ${ }^{32}, 2005$, Fleming et al. ${ }^{48}, 2005$, Mol$\operatorname{des}^{49}, 2003$, Mondelli $\left.{ }^{50}, 1999\right)$. Já nos casos de dentes bovinos, a origem dos elementos dentais não teve sua origem especificada (Yavuz et al. ${ }^{29}, 2006$, Braga et al. ${ }^{33}, 2006$, Calheiros et al. ${ }^{34}, 2004$, Sassi et al. ${ }^{35}, 2008$, Kawaguchi et al. ${ }^{36}, 2004$, Kubo et al. ${ }^{37}, 2004$, Silva Santana et al. ${ }^{39}$, 2009).

Atenção especial é necessária quando da utilização de dentes humanos obtidos em bancos de dentes. Essa origem atende integralmente todos os pressupostos éticos da utilização de dentes humanos, trabalhando em parceria com os Comitês de Ética em Pesquisa das instituições de ensino e pesquisa. Entretanto, os pesquisadores devem estar absolutamente atentos à forma como esses dentes são arma- zenados nos bancos de dentes, no que se refere à solução, temperatura e tempo de estocagem, sob pena de coletar amostra que tenha sido exposta a produtos que possam interferir nos resultados das pesquisas. Sendo assim, deve-se ter cautela ao selecionar os dentes provenientes de banco de dentes.

A técnica mais empregada para a limpeza dos dentes que irão compor a amostra foi a utilização de curetas, associada à profilaxia com pedra-pomes (Attam et al. ${ }^{4}, 2009$, Attar et al. ${ }^{5}, 2008$, Brackett et al. ${ }^{6}, 2004$, Celiberti e Lussi ${ }^{8}, 2005$, Cenci et al. ${ }^{9}, 2005$, Chuang et al. ${ }^{10}$, 2001, Delme et al. ${ }^{11}, 2005$, Elgalaid et al. ${ }^{12}$, 2004, Fugaro $^{13}, 2010$, Giachetti et al. ${ }^{14}, 2008$, Hegde et al. ${ }^{15}, 2009$, Helvatjoglu-Antoniades et al. ${ }^{16}, 2004$, Kucukesmen e Sonmez ${ }^{17}$, 2008, MR et al. ${ }^{18}, 2010$, Manhart et al. ${ }^{19}$, 2001, Nilgun Ozturk et al. ${ }^{21}, 2004$, Pradelle-Plasse et al..22, 2004, Yazici et al..24, 2001, Senawongse et al. ${ }^{25}, 2010$, Sharma et al. ${ }^{26}, 2009$, Sung et al. ${ }^{27}, 2004$, Wahab et al..68, 2003, Yavuz et al..99, 2006, Yazici et al. ${ }^{30}, 2008$, Youssef et al..1, 2006, Ziskind et al. ${ }^{32}, 2005$, Calheiros et al. ${ }^{34}$, 2004, Kawaguchi et al. ${ }^{36}, 2004$, Kubo et al. ${ }^{37}, 2004$, Silva Santana et al. ${ }^{39}, 2009$, Moldes $^{49}$, 2003), tendo sido também relatada a utilização do ultrassom (RP. et al. ${ }^{23}$, 2009, Koliniotou-Koumpia et al. ${ }^{51}$, 2004). Considera-se que ambas as técnicas são adequadas, devendo a escolha ser feita pelo pesquisador de acordo com suas conveniências e disponibilidade de equipamentos.

Durante o período de coleta de dentes para compor a amostra, alguns pesquisadores utilizam como meio de estocagem soluções de cloramina a $0,5 \%$, de timol a $0,1 \%$ ou hipoclorito de sódio a 2,6\% até a obtenção da amostra (Brackett et al. ${ }^{6}, 2004$, Celiberti e Lussi $^{8}, 2005$, Elgalaid et al. ${ }^{12}$, 2004, Giachetti et al. ${ }^{14}$, 2008, Helvatjoglu-Antoniades et al. ${ }^{16}, 2004$, Kucukesmen e Sonmez ${ }^{17}$, 2008, Manhart et al. ${ }^{19}, 2001$, Nilgun Ozturk et al. ${ }^{21}, 2004$, Pradelle-Plasse et al. ${ }^{22}, 2004$, Senawongse et al. ${ }^{25}, 2010$, Sharma et al. ${ }^{26}, 2009$, Yazici et al. ${ }^{30}, 2008$, Ziskind et al. ${ }^{32}, 2005$, Sassi et al. $.^{35}, 2008$, Fleming et al. $\left.{ }^{48}, 2005\right)$. Justifica-se a utilização dessas soluções para evitar crescimento bacteriano ou apenas
QUITERO MFZ

LOPES AO

MATOS $A B$

ENSAIO DE

MICROINFILTRAÇÃO: REVISÃO DA LITERATURA

125

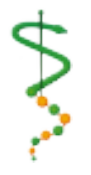

REV, ODONTOL.

UnIV. CID, SÃO PAULO

2012 ; $24(2)$ : 123 33, MAIO-AGO 
QUITERO MFZ LOPES AO MATOS $A B$

ENSAIO DE MICRO INFILTRAÇÃO: REVISÃO DA LITERATURA

REV, ODONTOL. UNIV, CID, SÃO PAULO 2012; 24(2): 12333. MAIO-AGO: para limpeza dos dentes. A estocagem em algumas soluções pode deixar resíduos e, de alguma maneira, interferir nos resultados obtidos. Dessa forma, ressalta-se que uma lavagem intensa ao final da estocagem é fundamental.

Findo esse período, os elementos dentais são armazenados em água destilada, solução salina ou saliva artificial até a montagem dos espécimes. Entre as etapas que compõem a metodologia, os espécimes mais freqüentemente utilizados ficam armazenados em água destilada em estufa até a conclusão do experimento.

\section{- Preparo cavitário e técnica restaura- dora}

Estudos de microinfiltração podem ser realizados em preparos cavitários de Classe II (Cenci et al. ${ }^{9}$, 2005, Chuang et al. ${ }^{10}, 2001$, MR et al. ${ }^{18}, 2010$, Yazici et al. ${ }^{24}, 2001$, Yazici et al. ${ }^{30}, 2008$, Ziskind et al. ${ }^{32}$, 2005, Fleming et al. ${ }^{48}$, 2005, Mondelli50, 1999, Bijjela ${ }^{52}$, 2000) ou V (Attam et al. ${ }^{4}, 2009$, Attar et al. ${ }^{5}, 2008$, Brackett et al. ${ }^{6}, 2004$, Delme et al. ${ }^{11}, 2005$, Fugaro $^{13}, 2010$, Giachetti et al. ${ }^{14}, 2008$, Hegde et al. ${ }^{15}, 2009$, Helvatjoglu-Antoniades et al. ${ }^{16}, 2004$, Kucukesmen e Sonmez ${ }^{17}$, 2008, Manhart et al. ${ }^{19}$, 2001, Nilgun Ozturk et al..21, 2004, RP. et al. ${ }^{23}, 2009$, Senawongse et al. ${ }^{25}, 2010$, Sharma et al..$^{26}$, 2009, Sung et al. ${ }^{27}, 2004$, Wahab et al. ${ }^{28}$, 2003, Yavuz et al. ${ }^{29}, 2006$, Calheiros et al. ${ }^{34}, 2004$, Sassi et al. $.^{35}, 2008$, Kawaguchi et al. ${ }^{36}, 2004$, Silva Santana et al. ${ }^{39}, 2009$, Moldes $^{49}, 2003$, Federici ${ }^{53}, 2009$, Nunes ${ }^{54}$, 2001, Pereira ${ }^{55}, 2007$, Tapety $\left.{ }^{56}, 2001\right)$.

Observa-se que a maioria dos trabaIhos utilizou cavidades Classe $\mathrm{V}$, realizadas principalmente no terço cervical dos dentes (Attam et al. ${ }^{4}, 2009$, Attar et al. ${ }^{5}$, 2008, Brackett et al. ${ }^{6}$, 2004, Delme et al. ${ }^{11}, 2005$, Giachetti et al. ${ }^{14}$, 2008, Hegde et al. ${ }^{15}$, 2009, Helvatjoglu-Antoniades et al. ${ }^{16}, 2004$, Kucukesmen e Sonmez ${ }^{17}$, 2008, Manhart et al. ${ }^{19}, 2001$, RP. et al..$^{23}$, 2009, Sharma et al. ${ }^{26}, 2009$, Yazici et al. ${ }^{30}$, 2008, Kawaguchi et al. ${ }^{36}, 2004$, Koliniotou-Koumpia et al. $\left.{ }^{51}, 2004\right)$, com ângulo cavossuperficial cervical localizado em esmalte (Attar et al. ${ }^{5}, 2008$, Brackett et al. ${ }^{6}, 2004$, Delme et al. ${ }^{11}, 2005$, Fugaro ${ }^{13}$, 2010, Hegde et al. ${ }^{15}$, 2009, Helvatjoglu-
-Antoniades et al. ${ }^{16}, 2004$, Kucukesmen e Sonmez ${ }^{17}$, 2008, Manhart et al. ${ }^{19}, 2001$, RP. et al. ${ }^{23}, 2009$, Sharma et al. ${ }^{26}, 2009$, Wahab et al. ${ }^{28}, 2003$, Yavuz et al. ${ }^{29}, 2006$, Yazici et al. ${ }^{30}, 2008$, Kawaguchi et al. ${ }^{36}$, 2004, Silva Santana et al.39, 2009, Koliniotou-Koumpia et al. $\left.{ }^{51}, 2004\right)$ ou dentina (Attar et al. ${ }^{5}, 2008$, Giachetti et al. ${ }^{14}$, 2008, Sassi et al. $\left..^{35}, 2008\right)$. As dimensões do preparo cavitário podem variar, porém as mais frequentes são de $2 \mathrm{~mm} X 3 \mathrm{~mm}$ $X 1,5 \mathrm{~mm}$ (altura $X$ largura $X$ profundidade) (Delme et al. ${ }^{11}, 2005$, Sharma et al. ${ }^{26}$, 2009, Yavuz et al. ${ }^{29}, 2006$, Yazici et al. ${ }^{30}$, 2008, Sassi et al. ${ }^{35}, 2008$, Kawaguchi et al. ${ }^{36}, 2004$, Koliniotou-Koumpia et al. ${ }^{51}$, 2004). Ressalta-se que os preparos cavitários podem ser realizados apenas na face vestibular Attam et al. ${ }^{4}, 2009$, Attar et al. ${ }^{5}, 2008$, Brackett et al. ${ }^{6}, 2004$, Delme et al. ${ }^{11}, 2005$, Hegde et al. ${ }^{15}, 2009$, Helvatjoglu-Antoniades et al. ${ }^{16}, 2004, \mathrm{Ku}-$ cukesmen e Sonmez ${ }^{17}$, 2008, Manhart et al. ${ }^{19}, 2001$, Nilgun Ozturk et al. ${ }^{21}, 2004$, Senawongse et al. ${ }^{25}, 2010$, Sung et al. ${ }^{27}$, 2004, Wahab et al. ${ }^{28}, 2003$, Calheiros et al. ${ }^{34}, 2004$, Sassi et al. ${ }^{35}, 2008$, Kawaguchi et al. ${ }^{36}, 2004$, Silva Santana et al..99, 2009, Koliniotou-Koumpia et al. ${ }^{51}, 2004$ ou com aproveitamento também da face lingual/ palatina dos dentes (Attar et al. ${ }^{5}, 2008$, Fugaro $^{13}$, 2010, Giachetti et al. ${ }^{14}, 2008$, RP. et al. $.^{23}, 2009$, Sharma et al. ${ }^{26}, 2009$, Yazici et al. $\left..^{30}, 2008\right)$.

Quanto às cavidades Classe II, verificou-se que foram realizadas nas faces mesiais e distais dos dentes, sendo o limite do preparo de 0,5 a $1,5 \mathrm{~mm}$ da junção esmalte-cemento (Chuang et al. ${ }^{10}, 2001$, Yazici et al. $.^{30}, 2008$, Ziskind et al. $\left.{ }^{32}, 2005\right)$, e foram realizados tanto preparos MOD (Yazici et al. $.^{30}, 2008$, Ziskind et al. $\left.{ }^{32}, 2005\right)$ quanto slots verticais (Casagrande et al. ${ }^{7}$, 2005, Cenci et al. ${ }^{9}, 2005$, Chuang et al. ${ }^{10}$, 2001). Nos preparos MOD, a largura da caixa oclusal foi de aproximadamente um terço da distância intercuspídica, e a largura da caixa proximal foi equivalente a um terço da distância vestíbulo-lingual (Yazici et al. ${ }^{30}$, 2008). Já nos slots, observou-se $4 \mathrm{~mm}$ de distância ocluso-lingual Chuang et al. ${ }^{10},(2001), 3 \mathrm{~mm}$ de distância ocluso-gengival Chuang et al. ${ }^{10}$, (2001) e $2 \mathrm{~mm}$ de profundidade (Cenci et al. $\left.{ }^{9}, 2005\right)$. 
Muito frequentemente, a forma de aplicar o sistema adesivo, de instalar dispositivos auxiliares ao procedimento restaurador, tais como uso de matrizes e cunhas, bem como as técnicas restauradoras, constituem os fatores de variação do trabalho em questão. Apesar disso, observou-se que a maioria dos trabalhos segue as instruções do fabricante dos materiais utilizados no estudo. Na maior parte dos trabalhos consultados, a resina composta é inserida no preparo cavitário, utilizando-se a técnica incremental (Attam et al. ${ }^{4}, 2009$, Attar et al. ${ }^{5}, 2008$, Brackett et al. ${ }^{6}, 2004$, Cenci et al. ${ }^{9}, 2005$, Delme et al. ${ }^{11}$, 2005, Fugaro ${ }^{13}$, 2010, Hegde et al. ${ }^{15}$, 2009, Helvatjoglu-Antoniades et al. ${ }^{16}, 2004$, Kucukesmen e Sonmez ${ }^{17}$, 2008, MR et al. ${ }^{18}, 2010$, Manhart et al. ${ }^{19}$, 2001, Sung et al. ${ }^{27}, 2004$, Youssef et al. ${ }^{31}$, 2006, Kawaguchi et al. ${ }^{36}$, 2004, Fleming et al. $\left.{ }^{48}, 2005\right)$. Terminada a restauração, os espécimes são frequentemente estocados em água destilada por 24 horas em estufa a $37^{\circ} \mathrm{C}$ (Attar et al. ${ }^{5}, 2008$, Brackett et al. ${ }^{6}, 2004$, Cenci et al. ${ }^{9}, 2005$, Delme et al. ${ }^{11}$, 2005, Elgalaid et al. ${ }^{12}$, 2004, Fugaro $^{13}, 2010$, Giachetti et al. ${ }^{14}, 2008$, Hegde et al. ${ }^{15}$, 2009, Helvatjoglu-Antoniades et al. ${ }^{16}, 2004$, Kucukesmen e Sonmez ${ }^{17}$, 2008, MR et al. ${ }^{18}, 2010$, Manhart et al. ${ }^{19}$, 2001, Sharma et al. ${ }^{26}, 2009$, Yavuz et al. ${ }^{29}$, 2006, Yazici et al. ${ }^{30}, 2008$, Youssef et al. ${ }^{31}$, 2006, Ziskind et al. ${ }^{32}$, 2005, Calheiros et al. ${ }^{34}, 2004$, Sassi et al. ${ }^{35}, 2008$, Kawaguchi et al. ${ }^{36}, 2004$, Kubo et al. ${ }^{37}, 2004$, Silva Santana et al. ${ }^{39}, 2009$, Fleming et al. ${ }^{48}$, 2005, Radovic et al. $\left.{ }^{57}, 2008\right)$. Entretanto, outros estudos realizam os procedimentos de acabamento e polimento imediatamente após a inserção da resina composta (Attam et al. ${ }^{4}, 2009$, Attar et al. ${ }^{5}, 2008$, Chuang et al. ${ }^{10}, 2001$, Giachetti et al. ${ }^{14}$, 2008, Yazici et al. ${ }^{24}, 2001$, Senawongse et al. ${ }^{25}, 2010$, Sung et al. ${ }^{27}, 2004$, Koliniotou-Koumpia et al. $\left.{ }^{51}, 2004\right)$.

Os procedimentos de acabamento e polimento das restaurações são realizados através de diferentes técnicas, com a utilização de diversos instrumentais, sendo o mais comum entre os trabalhos consultados a utilização de discos de lixa de granulação decrescente (Attam et al. ${ }^{4}, 2009$, Cenci et al. ${ }^{9}, 2005$, Chuang et al. ${ }^{10}, 2001$,
Delme et al. ${ }^{11}$, 2005, Fugaro $^{13}$, 2010, Hegde et al. ${ }^{15}, 2009$, Manhart et al. ${ }^{19}, 2001$, Yazici et al. ${ }^{24}, 2001$, Senawongse et al. ${ }^{25}$, 2010, Sharma et al. ${ }^{26}, 2009$, Yazici et al. ${ }^{30}$, 2008, Braga et al.33, 2006, Sassi et al. ${ }^{35}$ 2008, Kawaguchi et al. ${ }^{36}, 2004$, Fleming et al. ${ }^{48}, 2005$, Koliniotou-Koumpia et al. ${ }^{51}$, 2004). Determinados procedimentos de acabamento e polimento podem criar, também, gaps na interface material restaurador/dente e, assim, facilitar a microinfiltração (Brackett et al. ${ }^{41}$, 1997, Prati et al. ${ }^{58,}$ 1997). Vale ressaltar que independente da técnica e instrumental utilizado, o acabamento e polimento visam fazer coincidir a margem da restauração com o ângulo cavossuperficial do preparo cavitário. Para tanto, é fundamental a utilização de iluminação adequada e lentes de aumento.

\section{- Procedimentos laboratoriais de enve- Ihecimento dos espécimes}

A ciclagem térmica e a ciclagem mecânica têm sido incluídas como metodologias específicas de envelhecimento de espécimes nos estudos da microinfiltração, simulando mudanças térmicas e/ou estresse mastigatório que acontecem no ambiente intraoral, que podem diminuir a vida útil das restaurações.

Os protocolos mais comumente utilizados são:

- Ciclagem térmica: 500 ciclos de $5^{\circ}$ $\mathrm{C}$ a $55^{\circ} \mathrm{C}$, com imersão por $1 \mathrm{mi}-$ nuto e tempo de transferência entre os dois banhos de 10 segundos em cada temperatura (Attam et al. ${ }^{4}$, 2009, Attar et al., , 2008, Brackett et al. ${ }^{6}, 2004$, Celiberti e Lussi ${ }^{8}, 2005$, Chuang et al. ${ }^{10}, 2001$, Delme et al. ${ }^{11}$, 2005, Fugaro ${ }^{13}$, 2010, Giachetti et al. ${ }^{14}, 2008$, Hegde et al..$^{15}$, 2009, Kucukesmen e Sonmez ${ }^{17}$, 2008, MR et al. ${ }^{18}, 2010$, Manhart et al. ${ }^{19}, 2001$, Moshonov et al. ${ }^{20}$, 2005, Nilgun Ozturk et al..21, 2004, Pradelle-Plasse et al..22, 2004, RP. et al. ${ }^{23}, 2009$, Yazici et al. ${ }^{24}, 2001$, Sharma et al. ${ }^{26}, 2009$, Sung et al. ${ }^{27}$, 2004, Wahab et al..28, 2003, Yavuz et al. $.^{29}, 2006$, Yazici et al. ${ }^{30}, 2008$, Youssef et al. ${ }^{31}, 2006$, Ziskind et al. ${ }^{32}, 2005$, Sassi et al. ${ }^{35}, 2008$, Kawaguchi et al. ${ }^{36}, 2004$, Kubo et
QUITERO MFZ

LOPES AO

MATOS AB

ensaio de

MICROINFILTRAÇÃO: REVISÃO DA LITERATURA

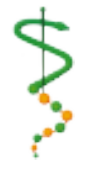

REV, ODONTOL.

UNIV, CID, SÃO PAULO

$2012 ; 24(2): 123-$ 33, MAIO-AGO 
QUITERO MFZ LOPES AO MATOS $A B$

ENSAIO DE MICROINFILTRAÇÃO: REVISÃO DA LITERATURA al. ${ }^{37}, 2004$, Silva Santana et al. ${ }^{39}$, 2009, Fleming et al.48, 2005).

- Ciclagem mecânica: frequência de 1,5 HZ por 250000 ciclos a $50 \mathrm{~N}$, com a força aplicada paralelamente ao longo do eixo do dente (Senawongse et al. ${ }^{25}$, 2010).

\section{- Protocolo de infiltração}

A impermeabilização dos espécimes é feita para evitar a entrada do corante por outro local além das margens da restauração. Os ápices dos dentes são selados e o dente impermeabilizado com 2 ou 3 camadas de esmalte cosmético, localizadas a $1 \mathrm{~mm}$ da margem da restauração. Após a aplicação de uma camada de esmalte, deve-se aguardar até sua completa secagem para a aplicação da camada subsequente.

A infiltração dos espécimes pode ser realizada através da utilização de corantes ou traçadores, escolhidos pelos pesquisadores conforme sua conveniência e treinamento para a técnica. Dentre os corantes mais utilizados, tem-se azul de metileno (Tabela 1) com concentrações que podem variar de $0,5 \%$ a $10 \%$, com tempo de imersão entre 1 a 24 horas; e Fucsina básica (Tabela 2) com a concentração variando entre $0,5 \%$ e $2 \%$, utilizada por 24 horas em temperatura ambiente. O traçador Nitrato de prata (Tabela 3) pode ser utilizado em concentração de $0,5 \%$ a $50 \%$ por 2 a 8 horas, no escuro, seguido de procedimento de revelação com luz.

Tabela 1 - Azul de metileno

\begin{tabular}{|c|c|}
\hline Concentração & Tempo de imersão \\
\hline $0,5 \%$ & 4h (Calheiros et al. $\left.{ }^{34}, 2004\right)$ \\
\hline $0,5 \%$ & $\begin{array}{l}\text { 24h (Casagrande et al. } \\
\text { 2005) }\end{array}$ \\
\hline $0,5 \%$ & $\begin{array}{l}\text { 3h (Senawongse et al. }{ }^{25} \text {, } \\
\text { 2010) }\end{array}$ \\
\hline $1 \%$ & $4 \mathrm{~h}\left(\right.$ Wahab et al. $\left.{ }^{28}, 2003\right)$ \\
\hline $1 \%$ & $\begin{array}{l}\text { 24h (Attam et al. }{ }^{4}, 2009 \\
\left.\text { Moshonov et al. }{ }^{20}, 2005\right)\end{array}$ \\
\hline $2 \%$ & $\begin{array}{l}\text { 24h (Delme et al. }{ }^{11}, 2005, \\
\text { Giachetti et al.14, 2008, } \\
\left.\text { Ziskind et al. } .^{32}, 2005\right)\end{array}$ \\
\hline $5 \%$ & 1h (Manhart et al. $\left.{ }^{19}, 2001\right)$ \\
\hline $5 \%$ & $\begin{array}{l}\text { 24h (Celiberti e Lussi }{ }^{8} \text {, } \\
\text { 2005) }\end{array}$ \\
\hline $10 \%$ & 4h (Brackett et al. $\left.{ }^{6}, 2004\right)$ \\
\hline
\end{tabular}

Tabela 2 - Fucsina básica

\begin{tabular}{|c|c|}
\hline Concentração & Tempo de imersão \\
\hline $0,2 \%$ & $\begin{array}{l}\text { 24h (Fleming et al. }{ }^{48} \text {, } \\
2005)\end{array}$ \\
\hline $0,5 \%$ & 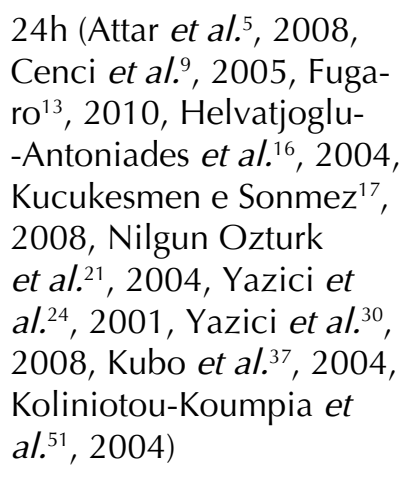 \\
\hline $2 \%$ & $\begin{array}{l}\text { 24h (Chuang et al. }{ }^{10}, 2001 \text {, } \\
\text { Helvatjoglu-Antoniades et } \\
\left.\text { al. }{ }^{16}, 2004\right)\end{array}$ \\
\hline
\end{tabular}

Tabela 3 - Nitrato de prata

\begin{tabular}{ll}
\hline \hline Concentração & Tempo de imersão \\
\hline $0,5 \%$ & $2 \mathrm{~h}$ (Braga et al. ${ }^{33}$, \\
& $2006)$ \\
$50 \%$ & $8 \mathrm{~h}\left(\right.$ Youssef et al..$^{31}$, \\
& 2006, Sassi et $a .^{35}$, \\
& 2008, Silva Santana \\
& et al. $\left.{ }^{39}, 2009\right)$ \\
$50 \%$ & $2 \mathrm{~h}\left(\mathrm{MR}\right.$ et al. ${ }^{18}$, \\
& $2010)$ \\
\hline \hline
\end{tabular}

O corante mais utilizado é o azul de metileno, pelo fato de ser extremamente solúvel e penetrar facilmente nos espaços dentais que contêm água Amarante de Camargo et al.59, 2006, além da simplicidade da técnica e de sua fácil obtenção. Entretanto, apresenta limitações, como o fato de perder sua cor ao entrar em contato com agentes redutores presentes em alguns materiais restauradores (Amarante de Camargo et al.59, 2006).

Quanto ao Nitrato de Prata, só é possível observar e medir sua infiltração utilizando-se um revelador radiográfico. Apesar desta dificuldade da técnica, sua penetração na dentina é maior quando comparada ao azul de metileno (Amarante de Camargo et al. ${ }^{59}, 2006$ ).

Assim, observa-se que nenhuma das duas abordagens é melhor que a outra, devendo a escolha estar baseada nas conveniências laboratoriais e na habilidade dos pesquisadores para uma ou outra técnica. 


\section{- Leitura dos resultados}

Os espécimes podem ser seccionados com um corte único (Attam et al. ${ }^{4}, 2009$, Attar et al. ${ }^{5}, 2008$, Brackett et al. ${ }^{6}, 2004$, Casagrande et al. ${ }^{7}, 2005$, Chuang et al. ${ }^{10}$, 2001, Delme et al. ${ }^{11}$, 2005, Fugaro ${ }^{13}$, 2010, Kucukesmen e Sonmez ${ }^{17}, 2008$, MR et al. ${ }^{18}, 2010$, Manhart et al. ${ }^{19}, 2001$, Nilgun Ozturk et al. $.^{21}, 2004$, RP. et al. ${ }^{23}$, 2009, Yazici et al. ${ }^{24}, 2001$, Sung et al. ${ }^{27}$, 2004, Wahab et al. ${ }^{28}, 2003$, Yavuz et al. ${ }^{29}$, 2006, Yazici et al. ${ }^{30}, 2008$, Youssef et al. ${ }^{31}$, 2006, Kawaguchi et al. ${ }^{36}, 2004$, Silva Santana et al. ${ }^{39}, 2009$, Brackett et al. ${ }^{41}$, 1997, Fleming et al. $\left.{ }^{48}, 2005\right)$ ou múltiplo (Celiberti e Lussi $^{8}, 2005$, Cenci et al. ${ }^{9}, 2005$, Giachetti et al. ${ }^{14}, 2008$, Hegde et al. ${ }^{15}$, 2009, Helvatjoglu-Antoniades et al. ${ }^{16}$, 2004, Manhart et al. ${ }^{19}$, 2001, Moshonov et al. ${ }^{20}, 2005$, Senawongse et al. ${ }^{25}, 2010$, Sharma et al. ${ }^{26}, 2009$, Braga et al. $.^{33}, 2006$, Calheiros et al. ${ }^{34}, 2004$, Sassi et al. ${ }^{35}, 2008$, Koliniotou-Koumpia et al.51, 2004, Prati et al. ${ }^{58}$, 1997, Amarante de Camargo et al. ${ }^{59}$, 2006). A escolha de que tipo utilizar deve ser do pesquisador, de acordo com o tipo de preparo cavitário realizado e com os fatores de variação em questão no estudo. O objetivo de realizar cortes múltiplos é a possibilidade de fazer mais análises em menores espaços onde estão o preparo cavitário e a restauração. O mais comum de encontrar é um corte único longitudinal no sentido vestíbulo-lingual no centro da restauração.

A leitura dos resultados pode realizar-se através de uma Análise Qualitativa, realizada através de escala de escores, que é a mais comumente utilizada (Attam et al. ${ }^{4}$, 2009, Attar et al. ${ }^{5}, 2008$, Brackett et al. ${ }^{6}$, 2004, Casagrande et al. ${ }^{7}, 2005$, Celiberti e Lussi ${ }^{8}, 2005$, Chuang et al. ${ }^{10}, 2001$, Fugaro $^{13}$, 2010, Giachetti et al. ${ }^{14}, 2008$, Hegde et al. ${ }^{15}$, 2009, Helvatjoglu-Antoniades et al. ${ }^{16}, 2004$, Kucukesmen e Sonmez ${ }^{17}$, 2008, MR et al. ${ }^{18}, 2010$, Manhart et al. ${ }^{19}$, 2001, Moshonov et al. ${ }^{20}$, 2005, Nilgun Ozturk et al. ${ }^{21}, 2004$, RP. et al. $.^{23}, 2009$, Yazici et al. ${ }^{24}, 2001$, Senawongse et al. ${ }^{25}$, 2010, Sharma et al. ${ }^{26}, 2009$, Sung et al. ${ }^{27}$, 2004, Wahab et al. ${ }^{28}, 2003$, Yavuz et al. ${ }^{29}$, 2006, Yazici et al. ${ }^{30}, 2008$, Youssef et al..$^{31}$, 2006, Ziskind et al. ${ }^{32}, 2005$, Kawaguchi et al. ${ }^{36}, 2004$, Kubo et al. ${ }^{37}, 2004$, Silva Santana et al. ${ }^{39}, 2009$, Fleming et al. ${ }^{48}, 2005$, Koliniotou-Koumpia et al. $\left.{ }^{51}, 2004\right)$. Apesar da facilidade da técnica, é fundamental que os observadores sejam calibrados. Outro modo de leitura é a Análise Quantitativa (morfométrica) (Cenci et al. ${ }^{9}, 2005$, Sassi et al. ${ }^{35}, 2008$, Pereira $\left.{ }^{55}, 2007\right)$. Neste caso, os cortes dentais são escaneados e as imagens obtidas são transferidas para um software que mede a extensão ou a área da penetração do corante ou traçador (Cenci et al. $\left.{ }^{9}, 2005\right)$

\section{CONCLUSÕES}

Conclui-se que muitas metodologias podem ser aplicadas em trabalhos de microinfiltração. A mais frequentemente encontrada na literatura utiliza dentes humanos com preparos cavitários de Classe V, tendo como método de envelhecimento a ciclagem térmica, o corante azul de metileno e a leitura dos resultados feita através da análise qualitativa. Os pesquisadores devem estar treinados e com condições de infraestrutura adequadas para o desenvolvimento da metodologia escolhida pelo grupo de trabalho.
QUITERO MFZ

LOPES AO

MATOS $A B$

ENSAIO DE

MICROINFILTRAÇÃO: REVISÃO DA

LITERATURA

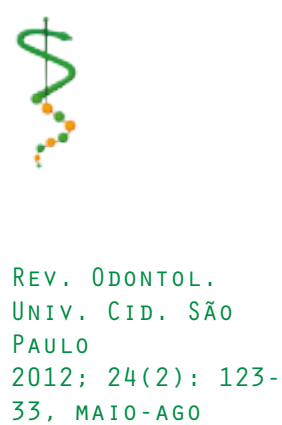


QUITERO MFZ

LOPES AO

MATOS $A B$

ENSAIO DE MICROINFILTRAÇÃO: REVISÃO DA LITERATURA

REV, ODONTOL. UNIV. CID. SÃO PAULO

\section{REFERÊNCIAS}

1. Kidd EA. Microleakage: a review. J Dent 1976 Sep;4(5):199-206.

2. Trowbridge HO. Model systems for determining biologic effects of microleakage. Oper Dent 1987 Autumn;12(4):164-72.

3. Akgungor G, Akkayan B. Influence of dentin bonding agents and polymerization modes on the bond strength between translucent fiber posts and three dentin regions within a post space. J Prosthet Dent 2006 May;95(5):368-78.

4. Attam K, Talwar S, Yadav S, Miglani S. Comparative analysis of the effect of autoclaving and $10 \%$ formalin storage on extracted teeth: A microleakage evaluation. J Conserv Dent 2009 Jan;12(1):26-30.

5. Attar N, Korkmaz Y, Ozel E, Bicer CO, Firatli E. Microleakage of class V cavities with different adhesive systems prepared by a diamond instrument and different parameters of Er:YAG laser irradiation. Photomed Laser Surg 2008 Dec;26(6):585-91.

6. Brackett WW, Haisch LD, Pearce MG, Brackett MG. Microleakage of Class V resin composite restorations placed with self-etching adhesives. J Prosthet Dent 2004 Jan;91(1):42-5.

7. Casagrande L, Brayner R, Barata JS, de Araujo FB. Cervical microleakage in composite restorations of primary teeth--in vitro study. J Dent 2005 Sep;33(8):627-32.

8. Celiberti P, Lussi A. Use of a self-etching adhesive on previously etched intact enamel and its effect on sealant microleakage and tag formation. J Dent 2005 Feb;33(2):16371.

9. Cenci M, Demarco F, de Carvalho R. Class II composite resin restorations with two polymerization techniques: relationship between microtensile bond strength and marginal leakage. J Dent 2005 Aug;33(7):603-10.

10. Chuang SF, Liu JK, Chao CC, Liao FP, Chen YH. Effects of flowable composite lining and operator experience on microleakage and internal voids in class II composite restorations. J Prosthet Dent 2001 Feb;85(2):177-83.

11. Delme KI, Deman PJ, De Moor RJ. Microleakage of class V resin composite restorations after conventional and Er:YAG laser preparation. J Oral Rehabil 2005 Sep;32(9):676-85.

12. Elgalaid TO, Youngson CC, McHugh S, Hall AF, Creanor SL, Foye RH. In vitro dentine permeability: the relative effect of a dentine bonding agent on crown preparations. J Dent 2004 Jul;32(5):413-21.

13. Fugaro OJ. COMMENTARY. Effect of prerestorative home-bleaching on microleakage of self-etch adhesives. J Esthet Restor Dent 2010 Jun;22(3):193.

14. Giachetti L, Scaminaci Russo D, Bambi C, Nieri M, Bertini F. Influence of operator skill on microleakege of total-etch and self-etch bonding systems. J Dent 2008 Jan;36(1):49-53.

15. Hegde MN, Vyapaka P, Shetty S. A comparative evaluation of microleakage of three different newer direct composite resins using a self etching primer in class $V$ cavities: An in vitro study. J Conserv Dent 2009 Oct;12(4):160-3.

16. Helvatjoglu-Antoniades M, Kalinderis K, Pedulu L, Papadogiannis Y. The effect of pulse activation on microleakage of a 'packable' composite resin and two 'ormocers'. J Oral Rehabil 2004 Nov;31(11):1068-74.

17. Kucukesmen C, Sonmez H. Microleakage of class-v composite restorations with different bonding systems on fluorosed teeth. Eur J Dent 2008 Jan;2(1):48-58. 
18. MR, Sajjan GS, B NK, Mittal N. Effect of different placement techniques on marginal microleakage of deep class-II cavities restored with two composite resin formulations. J Conserv Dent 2010 Jan;13(1):9-15.

19. Manhart J, Chen HY, Mehl A, Weber K, Hickel R. Marginal quality and microleakage of adhesive class V restorations. J Dent 2001 Feb;29(2):123-30.

20. Moshonov J, Stabholz A, Zyskind D, Sharlin E, Peretz B. Acid-etched and erbium:yttrium aluminium garnet laser-treated enamel for fissure sealants: a comparison of microleakage. Int J Paediatr Dent 2005 May;15(3):205-9.

21. Nilgun Ozturk A, Usumez A, Ozturk B, Usumez S. Influence of different light sources on microleakage of class V composite resin restorations. J Oral Rehabil 2004 May;31(5):500-4.

22. Pradelle-Plasse N, Wenger F, Picard B, Colon P. Evaluation of microleakage of composite resin restorations by an electrochemical technique: the impedance methodology. Dent Mater 2004 Jun;20(5):425-34.

23. RP., Bs S, Arunagiri D, Manuja N. Influence of hydrophobic layer and delayed placement of composite on the marginal adaptation of two self-etch adhesives. J Conserv Dent 2009 Apr;12(2):60-4.

24. Yazici AR, Frentzen M, Dayangac B. In vitro analysis of the effects of acid or laser etching on microleakage around composite resin restorations. J Dent 2001 Jul;29(5):355-61.

25. Senawongse P, Pongprueksa P, Tagami J. The effect of the elastic modulus of low-viscosity resins on the microleakage of Class $\mathrm{V}$ resin composite restorations under occlusal loading. Dent Mater J 2010 May;29(3):324-9.

26. Sharma V, Nainan MT, Shivanna V. The effect of cavity disinfectants on the sealing ability of dentin bonding system: An in vitro study. J Conserv Dent 2009 Jul;12(3):109-13.

27. Sung EC, Chan SM, Tai ET, Caputo AA. Effects of various irrigation solutions on microleakage of Class V composite restorations. J Prosthet Dent 2004 Mar;91(3):265-7.

28. Wahab FK, Shaini FJ, Morgano SM. The effect of thermocycling on microleakage of several commercially available composite Class $\mathrm{V}$ restorations in vitro. J Prosthet Dent 2003 Aug;90(2):168-74.

29. Yavuz I, Aydin H, Ulku R, Kaya S, Tumen C. A new method: measurement of microleakage volume using human, dog and bovine permanent teeth. Electronic Journal of Biotechnology 2006 Jan.;9(1):8-17.

30. Yazici AR, Celik C, Dayangac B, Ozgunaltay G. Effects of different light curing units/modes on the microleakage of flowable composite resins. Eur J Dent 2008 Oct;2(4):240-6.

31. Youssef MN, Youssef FA, Souza-Zaroni WC, Turbino ML, Vieira MM. Effect of enamel preparation method on in vitro marginal microleakage of a flowable composite used as pit and fissure sealant. Int J Paediatr Dent 2006 Sep;16(5):342-7.

32. Ziskind D, Adell I, Teperovich E, Peretz B. The effect of an intermediate layer of flowable composite resin on microleakage in packable composite restorations. Int J Paediatr Dent 2005 Sep;15(5):349-54.

33. Braga RR, Boaro LC, Kuroe T, Azevedo CL, Singer JM. Influence of cavity dimensions and their derivatives (volume and ' $C$ ' factor) on shrinkage stress development and microleakage of composite restorations. Dent Mater 2006 Sep;22(9):818-23.

QUITERO MFZ

LOPES AO

MATOS AB

ENSAIO DE

MICROINFILTRAÇÃO

REVISÃO DA

LITERATURA

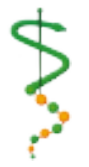

REV, OdONTOL.

Univ. CID. São

PAULO

2012; 24(2): 123 -

33, MAIO-AGO 
QUITERO MFZ

LOPES AO

MATOS $A B$

ENSAIO DE MICROINFILTRAÇÃO: REVISÃO DA LITERATURA

\section{2}

REV, ODONTOL :

UNIV. CID, SÃO

PAULO

2012; $24(2): 123$

33, MAIO-AGO
34. Calheiros FC, Sadek FT, Braga RR, Cardoso PE. Polymerization contraction stress of low-shrinkage composites and its correlation with microleakage in class $\mathrm{V}$ restorations. J Dent 2004 Jul;32(5):407-12.

35. Sassi JF, Batista AR, Ciccone-Nogueira JC, Corona SAM, Palma-Dibb RG. Influence of light-curing unit systems on shear bond strength and marginal microleakage of composite resin restorations. Mat Res 2008 jan.-mar.;11(1):69-73.

36. Kawaguchi FA, Eduardo CP, Matos AB. Nd:YAG laser influence on microleakage of class V composite restoration. Photomed Laser Surg 2004 Aug;22(4):303-5.

37. Kubo S, Yokota H, Hayashi Y. The effect of light-curing modes on the microleakage of cervical resin composite restorations. J Dent 2004 Mar;32(3):247-54.

38. Nakamichi I, Iwaku M, Fusayama T. Bovine teeth as possible substitutes in the adhesion test. J Dent Res 1983 Oct;62(10):1076-81.

39. Silva Santana SV, Bombana AC, Florio FM, Basting RT. Effect of surface sealants on marginal microleakage in Class $\mathrm{V}$ resin composite restorations. J Esthet Restor Dent 2009 21(6):397-404.

40. Brackett WW, Gunnin TD, Gilpatrick RO, Browning WD. Microleakage of Class $\mathrm{V}$ compomer and light-cured glass ionomer restorations. J Prosthet Dent 1998 Mar;79(3):261-3.

41. Brackett WW, Gilpatrick RO, Gunnin TD. Effect of finishing method on the microleakage of Class V resin composite restorations. Am J Dent 1997 Aug;10(4):189-91.

42. Schmalz G, Hiller KA, Nunez LJ, Stoll J, Weis K. Permeability characteristics of bovine and human dentin under different pretreatment conditions. J Endod 2001 Jan;27(1):23-30.

43. Nakabayashi N, Pashley D. Properties of dentin. Hibridização dos tecidos dentais duros. São Paulo: Quintessence; 2000.

44. Retief DH. Standardizing laboratory adhesion tests. Am J Dent 1991 Oct;4(5):231-6.

45. Schilke R, Lisson JA, Bauss O, Geurtsen W. Comparison of the number and diameter of dentinal tubules in human and bovine dentine by scanning electron microscopic investigation. Arch Oral Biol 2000 May;45(5):355-61.

46. Titley K, Chernecky R, Chan A, Smith D. The composition and ultrastructure of resin tags in etched dentin. Am J Dent 1995 Oct;8(5):224-30.

47. Efes B. Microleakage of composite materials in bovine and human teeth. J Dent Res 200382 Spec(Abstrac 1957):256.

48. Fleming GJ, Hall DP, Shortall AC, Burke FJ. Cuspal movement and microleakage in premolar teeth restored with posterior filling materials of varying reported volumetric shrinkage values. J Dent 2005 Feb;33(2):139-46.

49. Moldes V. Estudo in vitro da microinfiltração em restaurações de cavidades classe $\checkmark$ preparadas com alta rotação e lasers Er:YAG e Er, Cr:YSGG, utilizando-se dois sistemas adesivos [Tese]. São Paulo: Universidade de São Paulo; 2003.

50. Mondelli A. Avaliação da infiltração marginal em cavidades classe II restauradas com resina composta associada a outros materiais de inserção direta [Mestrado]. Bauru, SP: Faculdade de Odontologia de Bauru - USP; 1999.

51. Koliniotou-Koumpia E, Dionysopoulos P, Koumpia E. In vivo evaluation of microleakage from composites with new dentine adhesives. J Oral Rehabil 2004 Oct;31(10):1014-22. 
52. Bijjela M. Avaliação in vitro da microinfiltração marginal em restaurações classe II, confeccionadas com um cimento de ionômero de vidro modificado por resina, uma resina composta "semicondensavel" e uma resina composta "condensável" [Mestrado]. Bauru, SP: Faculdade de Odontologia de Bauru - USP; 2000.

53. Federici B. Importância de três técnicas de preparo cavitário e de três sistemas adesivos na microinfiltração marginal em restaurações Classe $\mathrm{V}$ de resina composta [Mestrado]. São Paulo: Universidade de São Paulo; 2009.

54. Nunes OBCN. Avaliação "in vitro" da microinfiltração marginal em cavidades de Classe $\mathrm{V}$, restauradas com resinas compostas condensáveis, resina composta híbrida, resina composta modificada por poliácidos e ionômero de vidro modificado por resina [Tese]. Bauru, SP: Faculdade de Odontologia de Bauru - USP; 2001.

55. Pereira AFVP. Microinfiltração e adaptação marginal de restaurações classe $V$ simulando lesões de abfração [Doutorado]. São Paulo: Universidade de São Paulo; 2007.

56. Tapety CMCT. Influência da interposição de bases na infiltração marginal em cavidades classe II (MOD), restauradas com resinas compostas para dentes posteriores [Mestrado]. Bauru, SP: Faculdade de Odontologia de Bauru - USP; 2001.

57. Radovic I, Mazzitelli C, Chieffi N, Ferrari M. Evaluation of the adhesion of fiber posts cemented using different adhesive approaches. Eur J Oral Sci 2008 Dec;116(6):55763.

58. Prati C, Chersoni S, Cretti L, Mongiorgi R. Marginal morphology of Class V composite restorations. Am J Dent 1997 Oct;10(5):231-6.

59. Amarante de Camargo DA, Sinhoreti MA, Correr-Sobrinho L, de Sousa Neto MD, Consani S. Influence of the methodology and evaluation criteria on determining microleakage in dentin-restorative interfaces. Clin Oral Investig 2006 Dec;10(4):31723.

Recebido em: 23/04/2011

Aceito em: 15/09/2011

QUITERO MFZ

LOPES AO

MATOS $A B$

ENSAIO DE

MI CROINFILTRAÇÃO:

REVISÃO DA

LITERATURA

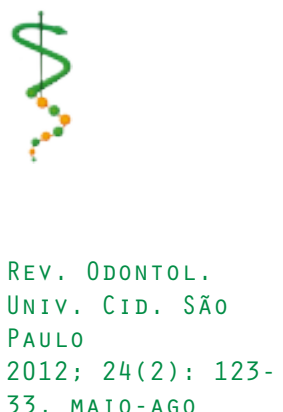

